\title{
Tuberculose no sistema prisional de Campinas, São Paulo, Brasil
}

\author{
Helenice Bosco de Oliveira' e Janaina Corrêa Cardoso²
}

Como citar Oliveira HB de, Cardoso JC. Tuberculose no sistema prisional de Campinas, São Paulo, Brasil. Rev Panam Salud Publica. 2004;15(3):194-9.

RESUMO Objetivo. Descrever a situação da tuberculose nas prisões da Cidade de Campinas, Estado de São Paulo, Brasil.

Métodos. Realizou-se um estudo retrospectivo dos casos notificados de tuberculose em presidiários no período de 1993 a 2000. Foram avaliados dados de 4293 detentos dos quatro presídios que constituem o complexo penitenciário da região. Foram utilizadas informações do banco de dados em tuberculose do Departamento de Medicina Preventiva e Social da Faculdade de Ciências Médicas da Universidade Estadual de Campinas (UNICAMP).

Resultados. Observou-se um pico de incidência da tuberculose em 1994 (1 397,62 notificações em 100000 indivíduos). A menor taxa foi observada em 1999 (559,04). Predominou a faixa etária de 25 a 34 anos $(62,6 \%)$ e a AIDS como doença associada (49,9\%). A forma preponderante de tuberculose foi a pulmonar (91,9\%), com baciloscopia de escarro positiva em 70,3\%. A maioria dos presidiários não tinha realizado tratamentos prévios (75,4\%). O abandono de tratamento chegou a 49\%. Houve cura em apenas 20,8\% dos casos no período estudado. Conclusões. A incidência intermediária de tuberculose observada neste estudo pode estar relacionada à subnotificação. A freqüência de falência do tratamento ainda é baixa. No entanto, o abandono, como fator que facilita a propagação de micobactérias resistentes na comunidade, causa preocupação. O controle da tuberculose nas prisões deveria ser parte rotineira das intervenções direcionadas a eliminar a doença na comunidade e a prevenir a resistência às drogas antituberculose.

Palavras-chave Epidemiologia, prisões, tuberculose.

Em todo o mundo, mas especialmente nos países em desenvolvimento, a epidemia de AIDS está provocando profundo impacto no controle da tu-

\footnotetext{
1 Universidade Estadual de Campinas (UNICAMP), Faculdade de Ciências Médicas, Departamento de Medicina Preventiva e Social, Campinas (SP), Brasil. Correspondência e pedidos de separatas devem ser encaminhados a esta autora no seguinte endereço: Rua Waldir Aparecido da Silva 60, Condomínio Barão do Café, CEP 13083-065, Campinas, SP, Brasil. E-mail: helenice@unicamp.br

2 UNICAMP, Faculdade de Medicina, Campinas, SP, Brasil.
}

berculose. Atualmente, o HIV é o fator de risco mais importante para a progressão da infecção para doença tuberculosa (1). Além disso, a presença da tuberculose no sistema prisional tem sido descrita como uma ameaça: sem o controle da tuberculose nas prisões, sugere-se que não seria possível controlar a doença fora delas. Assim, as penitenciárias são importantes na origem e transmissão da tuberculose (2-5).

A incidência de tuberculose é maior entre os presos do que na população geral (5). Mesmo antes da prisão, muitos detentos estão expostos a fatores de alto risco para a doença, como desnutrição, higiene escassa e condições de vida inadequadas $(2,6)$. Além disso, as penitenciárias impõem um risco adicional para a transmissão: a superlotação (7) e a pouca ventilação são dois fatores que freqüentemente coexistem nas prisões, juntamente com condições sanitárias adversas, baixo nível socioeconômico e uso de drogas (3). O tempo de permanência na de- 
tenção também está associado ao aumento da infecção pela micobactéria (5). As populações das prisões têm, ainda, um risco aumentado para a coinfecção tuberculose/HIV que implica um risco anual de desenvolver a doença, semelhante ao risco vitalício estimado para indivíduos infectados apenas pelo bacilo de Koch (8).

Nas prisões, a principal fonte de transmissão da tuberculose são os pacientes com bacilos ao exame direto da expectoração (9). Ainda que a bacterioscopia positiva antes do tratamento se correlacione com um alto grau de infecciosidade, o mesmo não ocorre com o paciente sob tratamento, pela ação rápida e poderosa dos medicamentos. Entretanto, o descumprimento dos esquemas terapêuticos, o abandono do tratamento e os baixos índices de cura estão fazendo emergir a resistência aos tuberculostáticos como ameaça para a tentativa de controle (10). Em algumas prisões da antiga União Soviética, a mortalidade por tuberculose atingiu $24 \%$ (2). Embora a estratégia de tratamento de curta duração diretamente observado (DOTS) tenha sido comprovada como tecnicamente factível em prisões (2), altas taxas de multirresistência a drogas antituberculose e baixas taxas de cura têm sido documentadas (11).

O impacto da tuberculose nos presídios não se limita aos detentos - afeta também a comunidade com que se relacionam, ou seja, familiares e funcionários dos presídios, durante e após a detenção. Os muros são irrelevantes diante da trivialidade do movimento de indivíduos entre o sistema prisional e a comunidade. A infecção por tuberculose contraída na comunidade pode iniciar uma epidemia dentro de uma prisão, enquanto que a rota inversa de transmissão é igualmente possível. Além das freqüentes transferências, a demora na identificação de presos suspeitos de apresentar tuberculose e o descuido nos procedimentos de controle e terapia têm favorecido a eclosão da doença em prisões $(4,11)$.

Assim, o objetivo do presente trabalho foi descrever aspectos da epidemiologia da tuberculose no sistema prisional de Campinas, Estado de São
Paulo, Brasil, entre 1993 a 2000. Foram avaliados o coeficiente anual de incidência de tuberculose nos presídios e suas variações no período estudado, assim como a presença de co-infecção por HIV e o resultado do tratamento da tuberculose entre os presidiários.

\section{MATERIAIS E MÉTODOS}

Foi realizada uma análise retrospectiva (1993 a 2000) dos casos notificados de tuberculose entre presidiários na Cidade de Campinas. A população estudada foi de aproximadamente 4293 detentos por ano, com variações diárias, nos quatro presídios que constituem o complexo penitenciário da região.

Foram utilizadas as informações do Banco de Dados em Tuberculose do Departamento de Medicina Preventiva e Social da Faculdade de Ciências Médicas da Universidade Estadual de Campinas (UNICAMP). O banco de dados agrega as informações registradas nas fichas de notificação de casos de tuberculose, cujo preenchimento é obrigatório em todos os serviços de saúde. A principal fonte de informação para o preenchimento das fichas é o prontuário clínico do paciente. As fichas contêm informações sobre tratamentos anteriores para tuberculose, forma clínica, processo de descoberta do caso e tempo decorrido até o início do tratamento; resultados de baciloscopia, raios- $X$ e outros exames; doenças associadas; e dados sobre internação. A digitação do conteúdo das fichas no banco de dados é feita rotineiramente, segundo coortes anuais, pela data de início de tratamento. É importante ressaltar que o preenchimento das fichas, na rotina, foi avaliado como precário.

Os pacientes foram estudados quanto à realização de tratamentos anteriores, forma clínica da tuberculose, resultados de baciloscopia de escarro, presença da co-infecção por HIV e resultado do tratamento. $\mathrm{O}$ teste tuberculínico não é utilizado como método diagnóstico complementar na admissão dos detentos no sistema prisional.
Foram classificados como virgens de tratamento os presos que nunca haviam recebido tratamento para tuberculose. As recidivas indicaram tratamento anterior com alta por cura e um período de latência antes do aparecimento de novo episódio da doença. Os indivíduos com história de abandono anterior foram aqueles que não completaram o esquema terapêutico, tendo abandonado o tratamento e posteriormente reingressado. Para cálculo de incidência foi considerada a população real dos presídios (4 293 detentos), uma vez que esta ultrapassava, em muito, a capacidade dos mesmos (2 910 vagas).

O esquema terapêutico empregado para tratamento da tuberculose nos presídios consistiu do uso diário, autoadministrado, de rifampicina e isoniazida por 6 meses, com pirazinamida diária durante os 2 primeiros meses. Não foram realizadas provas de sensibilidade em pacientes com tratamento anterior para tuberculose. Em relação ao teste anti-HIV, não foi possível saber se os pacientes deram seu consentimento informado para a realização do teste. A maior parte dos pacientes com AIDS já apresentava a doença por ocasião do tratamento da tuberculose. Outros detentos, pela gravidade do caso, foram diagnosticados durante a hospitalização, fora dos presídios.

O programa Epi Info (versão 6) foi utilizado para análise dos dados (12).

\section{RESULTADOS}

A tabela 1 mostra a distribuição dos presidiários com tuberculose segundo ano de início do tratamento. A idade predominante foi de 25 a 34 anos $(62,6 \%)$ em todos os anos estudados (tabela 2). A escolaridade foi baixa, no máximo 8 anos de estudo. Apenas $73,6 \%$ dos presos realizaram teste para detecção do HIV (tabela 3) e, dentre estes, $67,8 \%$ estavam infectados pelo vírus. A AIDS foi diagnosticada em $49,9 \%$ dos presos com tuberculose.

A forma pulmonar foi a mais freqüente (91,9\%). Muitos presos tinham mais de um órgão acometido. Nas for- 
TABELA 1. Presidiários com tuberculose segundo ano de início do tratamento, Campinas (SP), Brasil, 1993 a $2000^{a}$

\begin{tabular}{|c|c|c|c|c|c|c|c|c|c|c|c|c|c|c|c|c|c|c|}
\hline & \multicolumn{18}{|c|}{ Início do tratamento } \\
\hline & \multicolumn{2}{|c|}{1993} & \multicolumn{2}{|c|}{1994} & \multicolumn{2}{|c|}{1995} & \multicolumn{2}{|c|}{1996} & \multicolumn{2}{|c|}{1997} & \multicolumn{2}{|c|}{1998} & \multicolumn{2}{|c|}{1999} & \multicolumn{2}{|c|}{2000} & \multicolumn{2}{|c|}{ Total } \\
\hline & No. & $\%$ & No. & $\%$ & No. & $\%$ & No. & $\%$ & No. & $\%$ & No. & $\%$ & No. & $\%$ & No. & $\%$ & No. & $\%$ \\
\hline Casos & 33 & 9,2 & 60 & 16,7 & 45 & 12,5 & 48 & 13,4 & 51 & 14,2 & 51 & 14,2 & 24 & 6,7 & 47 & 13,1 & 359 & 100 \\
\hline Incidência & \multicolumn{2}{|c|}{768,69} & \multicolumn{2}{|c|}{1397,62} & \multicolumn{2}{|c|}{1048,21} & \multicolumn{2}{|c|}{1118,09} & \multicolumn{2}{|c|}{1187,98} & \multicolumn{2}{|c|}{1187,98} & \multicolumn{2}{|c|}{559,04} & \multicolumn{2}{|c|}{1094,80} & & \\
\hline
\end{tabular}

a População total de presidiários $=4293$

mas pulmonares, 70,3\% apresentaram baciloscopia de escarro positiva (tabela 4). Verificou-se que a maioria dos presos não sabia ou não tinha realizado tratamentos anteriores $(75,4 \%)$ (tabela 5).

Não foram obtidas informações sobre o resultado do tratamento para 114 pacientes. Para aqueles com disponibilidade do dado, verificou-se altas taxas de abandono (49,0\%) (tabela 6) e apenas uma falência de tratamento. Dentre os 42 presidiários que foram a óbito, 85,7\% tinham AIDS, e igual percentual não havia realizado tratamento anteriormente.

\section{DISCUSSÃO}

Os institutos correcionais, tradicionalmente, não têm sido considerados como participantes importantes nas intervenções de saúde pública (13). A tuberculose nas prisões já foi rotulada como uma praga esquecida (14), e as inúmeras ações preconizadas para o controle da tuberculose nesse ambiente (15) não são rotineiramente adotadas, evidenciando descaso e negligência. Atividades como a busca ativa de casos, a utilização da cultura perante baciloscopias negativas com imagem radiológica suspeita, a solici- tação de baciloscopias de escarro para controle de tratamento e o oferecimento de teste para detecção do HIV foram atividades intermitentes na rotina de controle da tuberculose nas prisões estudadas.

A ocupação dos presídios em Campinas excedeu em quase $50 \%$ a capacidade dessas instituições, facilitando a transmissão. Deve-se considerar que muitos detentos podem ter sido infectados pelo bacilo antes de ingressarem no sistema penitenciário, e que outros podem ter sido infectados na prisão e saído do sistema sem ter diagnosticada a tuberculose. O número de pes-

TABELA 2. Presidiários com tuberculose segundo ano de início do tratamento e faixa etária, Campinas (SP), Brasil, 1993 a 2000

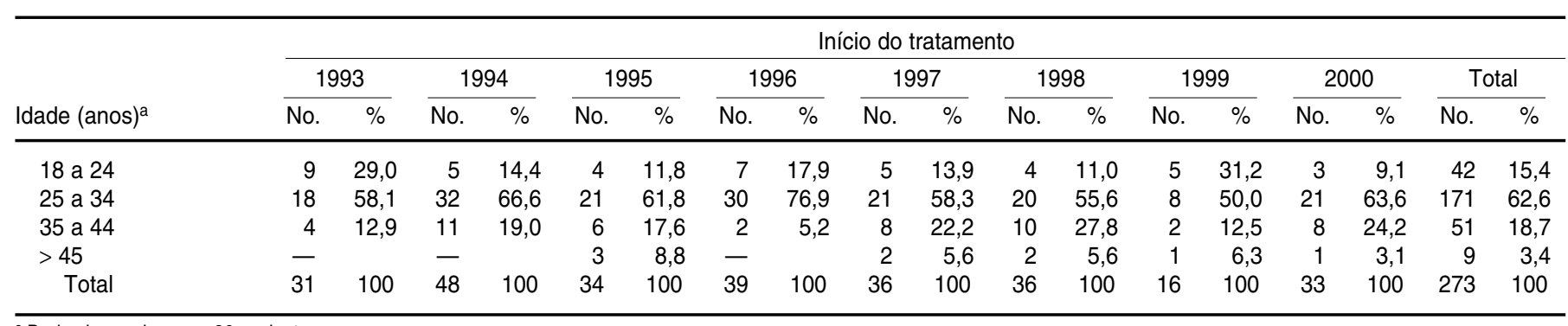

${ }^{a}$ Dados ignorados para 86 pacientes.

TABELA 3. Presidiários com tuberculose segundo ano de início do tratamento e sorologia para HIV, Campinas (SP), Brasil, 1993 a 2000

\begin{tabular}{|c|c|c|c|c|c|c|c|c|c|c|c|c|c|c|c|c|c|c|}
\hline \multirow[b]{3}{*}{ Status HIV } & \multicolumn{18}{|c|}{ Início do tratamento } \\
\hline & \multicolumn{2}{|c|}{1993} & \multicolumn{2}{|c|}{1994} & \multicolumn{2}{|c|}{1995} & \multicolumn{2}{|c|}{1996} & \multicolumn{2}{|c|}{1997} & \multicolumn{2}{|c|}{1998} & \multicolumn{2}{|c|}{1999} & \multicolumn{2}{|c|}{2000} & \multicolumn{2}{|c|}{ Total } \\
\hline & No. & $\%$ & No. & $\%$ & No. & $\%$ & No. & $\%$ & No. & $\%$ & No. & $\%$ & No. & $\%$ & No. & $\%$ & No. & $\%$ \\
\hline Negativo & 12 & 36,4 & 12 & 20,0 & 7 & 15,6 & 10 & 20,8 & 16 & 31,4 & 13 & 25,5 & 4 & 16,7 & 11 & 23,4 & 85 & 23,7 \\
\hline Não realizado & 8 & 24,2 & 21 & 35,0 & 11 & 24,4 & 6 & 12,5 & 10 & 19,6 & 10 & 19,6 & 11 & 45,8 & 18 & 38,3 & 95 & 26,4 \\
\hline Total & 33 & 100 & 60 & 100 & 45 & 100 & 48 & 100 & 51 & 100 & 51 & 100 & 24 & 100 & 47 & 100 & 359 & 100 \\
\hline
\end{tabular}


TABELA 4. Baciloscopia de escarro entre presidiários com tuberculose pulmonar conforme ano de início do tratamento, Campinas (SP), Brasil, 1993 a 2000

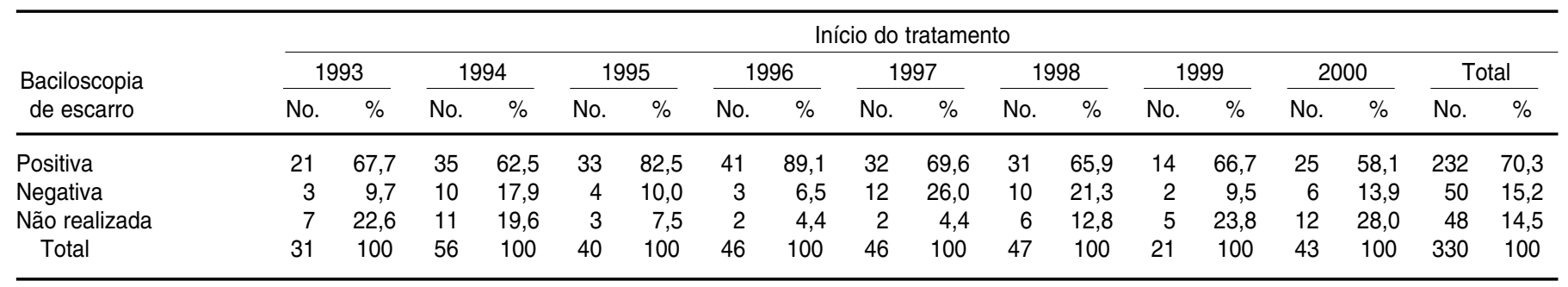

TABELA 5. Presidiários com tuberculose segundo ano de início do tratamento e tratamentos prévios, Campinas (SP), Brasil, 1993 a 2000

\begin{tabular}{|c|c|c|c|c|c|c|c|c|c|c|c|c|c|c|c|c|c|c|}
\hline \multirow[b]{3}{*}{ Tratamento prévio ${ }^{a}$} & \multicolumn{18}{|c|}{ Início do tratamento } \\
\hline & \multicolumn{2}{|c|}{1993} & \multicolumn{2}{|c|}{1994} & \multicolumn{2}{|c|}{1995} & \multicolumn{2}{|c|}{1996} & \multicolumn{2}{|c|}{1997} & \multicolumn{2}{|c|}{1998} & \multicolumn{2}{|c|}{1999} & \multicolumn{2}{|c|}{2000} & \multicolumn{2}{|c|}{ Total } \\
\hline & No. & $\%$ & No. & $\%$ & No. & $\%$ & No. & $\%$ & No. & $\%$ & No. & $\%$ & No. & $\%$ & No. & $\%$ & No. & $\%$ \\
\hline Não sabe/não tratou & 24 & 72,7 & 51 & 85,0 & 36 & 81,8 & 35 & 72,9 & 27 & 52,9 & 39 & 78,0 & 17 & 77,3 & 38 & 82,6 & 267 & 75,4 \\
\hline Recidiva & 3 & 9,1 & 4 & 6,7 & 2 & 4,5 & 6 & 12,5 & 6 & 11,8 & 4 & 8,0 & $\ldots$ & & 2 & 4,3 & 27 & 7,6 \\
\hline Abandono & 6 & 18,2 & 5 & 8,3 & 6 & 13,7 & 7 & 14,6 & 18 & 35,3 & 7 & 14,0 & 5 & 22,7 & 5 & 10,9 & 59 & 16,7 \\
\hline Falência & - & & - & & - & & - & & - & & - & & - & & 1 & 2,2 & 1 & 0,3 \\
\hline Total & 33 & 100 & 60 & 100 & 44 & 100 & 48 & 100 & 51 & 100 & 50 & 100 & 22 & 100 & 46 & 100 & 354 & 100 \\
\hline
\end{tabular}

a Dados ignorados para cinco pacientes.

soas expostas no presídio e que têm seu diagnóstico feito meses ou anos depois não pode ser determinado, podendo ser substancial $(13,16)$.

Valway et al. (17) afirmam que a demora em identificar e isolar sintomáticos ou suspeitos de terem tuberculose, as freqüentes transferências, as falhas nos procedimentos de controle de infecções e as inadequações no tratamento são fatores que contribuem para a presença da tuberculose nas prisões.
Observam, ainda, que as transferências de detentos entre prisões são comuns em todos os sistemas, e que as avaliações de saúde não são feitas rotineiramente antes da transferência.

A incidência da tuberculose no sistema penitenciário em Campinas variou de 1397,62 em 100000 indivíduos (em 1994) a 559,04 (em 1999). Tratase de uma incidência intermediária quando comparada a outros estudos: 2 775, conforme March et al. (4) e 5995 , conforme Aerts et al. (18). Não foi observada uma tendência definida de crescimento ou redução, sendo que as taxas observadas podem estar refletindo falhas na notificação, bem como demora na detecção de novos casos, problemas agravados por várias rebeliões durante o período. Entre 1993 e 2000, a incidência notificada de tuberculose na população geral, na região onde se localizam os presídios, esteve ao redor de 50 em 100000 indivíduos, e

TABELA 6. Presidiários com tuberculose segundo ano de início do tratamento e resultado do tratamento realizado, Campinas (SP), Brasil, 1993 a 2000

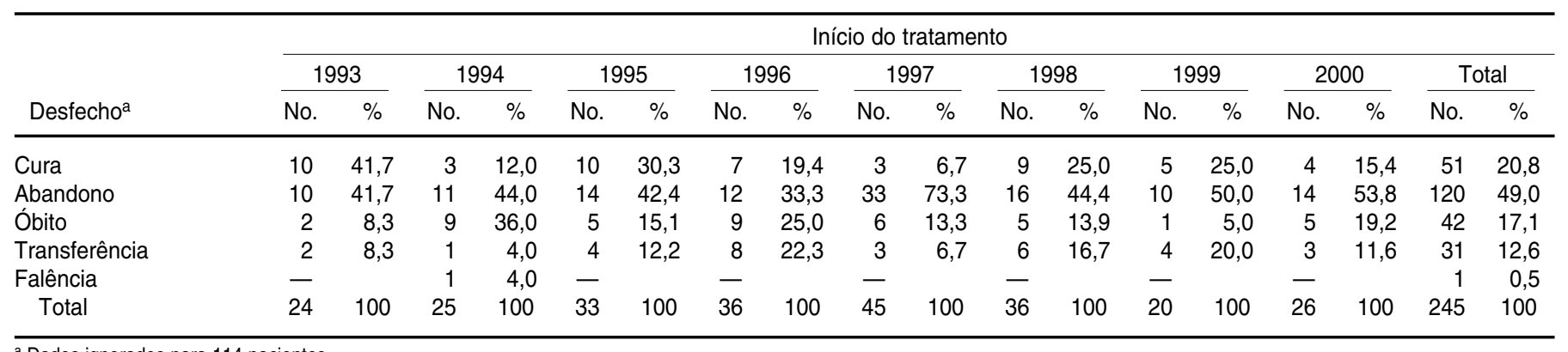

a Dados ignorados para 114 pacientes. 
a prevalência de HIV entre doentes de tuberculose ficou entre 10 e 15\% (19).

Em Campinas, predominaram detentos entre 25 e 34 anos, conforme relatado em outros estudos (13, 16, 18, 20). Além disso, a tuberculose esteve intimamente relacionada à baixa escolaridade. Um estudo na Espanha (20) constatou que $53,8 \%$ dos presos não obtiveram certificado de conclusão da educação primária.

Neste estudo, a AIDS foi a principal doença associada à tuberculose. As taxas de prevalência da AIDS citadas por outros autores variam: na Espanha, em 2000, March et al. (4) encontraram $85 \%$, contra $17,5 \%$ relatados por estudo anterior (20) no mesmo país; nos Estados Unidos, Jones et al. relatam uma prevalência de 13\% de AIDS em 1999 (13). Também no presente estudo, a forma clínica pulmonar da tuberculose esteve presente na maioria dos casos. Tal dado é corroborado por outros investigadores $(4,16)$. A positividade da baciloscopia $(70,3 \%)$ foi superior àquela relatada por outros estudos, que variou entre 29 e $41 \%(4,13,16)$.

Em Campinas, não foi possível obter informações acerca do encerramento do tratamento para $114(31,8 \%)$ pacientes, mostrando a precariedade da vigilância da tuberculose no sistema penitenciário. Dentre os presos cujos dados estavam disponíveis, apenas 20,8\% foram considerados curados. Em estudo no Azerbaijão, Coninx et al.
(11) observaram que $54 \%$ dos pacientes da população de estudo foram considerados curados após o tratamento, enquanto $13 \%$ não completaram o esquema e $11 \%$ foram a óbito.

O abandono do tratamento contribui de modo significativo para a grande transmissibilidade do bacilo nos presídios, além de contribuir para o aumento da resistência às diversas drogas $(11,21)$ e da propagação dessas micobactérias resistentes na comunidade $(11,17,22)$. Nos casos em que foi aplicado o tratamento supervisionado, não houve abandono e a cura foi alcançada na maioria dos pacientes, sendo a supervisão aconselhada em populações com alta taxa de resistência (11) ou com falhas no uso da medicação (17).

Usualmente não deveria haver morte por tuberculose: ainda que seja inadequado, o tratamento evita o óbito e mantém o indivíduo bacilífero e crônico $(21,23)$. O óbito ocorre tão somente se há demora ou falha no diagnóstico $(24,25)$. O atraso na instituição do tratamento é desastroso quando o paciente em questão é imunocomprometido (24). Estudos sobre cepas de Mycobacterium tuberculosis mostraram que a presença de infecção por HIV é um fator de risco para o desenvolvimento de resistência às drogas antituberculose (4). Uma terapia adequada possibilita a cura da tuberculose em pacientes com AIDS, prolongando a sobrevida (24).

\section{REFERÊNCIAS}

1. Pitchenik AE. Tuberculosis control and the AIDS epidemic in developing countries. Ann Intern Med. 1990;113(2):89-91.

2. Reyes H, Coninx R. Pitfalls of tuberculosis programmes in prisons. BMJ. 1997;315(7120): 1447-50.

3. Glaser JB, Greifinger RB. Correctional health care: a public health opportunity. Ann Inter Med. 1993;118(2):139-45.

4. March F, Coll P, Guerrero RA, Busquets E, Caylà JA. Predictors of tuberculosis transmission in prisons: an analysis using conventional and molecular methods. AIDS. 2000; 14(15):525-35.

5. Bellin EY, Fletcher DD, Safyer SM. Association of tuberculosis infection with increased time in or admission to the New York City jail system. JAMA. 1993;269(17):2228-31.
6. Martín V, González P, Caylà JA, Mirabent J, Canellas J, Pina JM, et al. Case-finding of pulmonary tuberculosis on admission to a penitentiary center. Tuberc Lung Dis. 1994;75(1): 49-53.

7. MacIntyre CR, Kendig N, Kummer L, Birago S, Graham NM. Impact of tuberculosis control measures and crowding on the incidence of tuberculosis infection in Maryland prisons. Clin Infect Dis. 1997;24(6):1060-7.

8. Styblo K. Epidemiology of tuberculosis. Selected papers. The Hague: Royal Netherlands Tuberculosis Association (KNCV); 1991.

9. Rouillon A, Perdrizet S, Parrot R, Waaler $H$. La transmisión del bacilo tuberculoso. El efecto de la quimioterapia. Em: Métodos de control de la tuberculosis. Washington, D.C.:
A estratégia do DOTS tem sido efetiva para a prevenção de novos casos, especialmente em áreas grandemente afetadas pelo $\operatorname{HIV}(4,26)$, juntamente com a busca ativa de casos e o diagnóstico precoce (27). Rodrigo et al. (28) constataram a redução da incidência da tuberculose nas prisões como resultado da implementação de tratamento supervisionado, da busca ativa de casos e da diminuição do tempo de demora do diagnóstico e do abandono de tratamento.

O monitoramento da tuberculose nas prisões deveria ser parte rotineira das intervenções direcionadas a eliminar a doença na comunidade. A cooperação entre o corpo médico das prisões e os programas de tuberculose na saúde pública é importante para assegurar o tratamento adequado e o seguimento dos pacientes quando eles se movem entre as prisões e a comunidade $(12,29)$. É imperativo vencer as dificuldades organizacionais, logísticas e políticas para que seja factível a aplicação da estratégias como o DOTS nas prisões, assegurando uma melhor qualidade de vida para os presos e um controle mais efetivo da tuberculose dentro e fora das prisões.

Agradecimentos. Os autores agradecem à Dra. Leticia Marin-León pela sugestões metodológicas e de organização do texto.
Organización Panamericana de la Salud; 1977. (Publicación Científica 346).

10. Kimerling ME, Kluge H, Vezhnina N, Iacovazzi $T$, Demeulenaere $T$, Portaels $F$, et al. Inadequacy of the current WHO re-treatment regimen in a central Siberian prison: treatment failure and MDR-TB. Int J Tuberc Lung Dis. 1999;3(5):451-3.

11. Coninx R, Mathieu C, Debacker M, Mirzoev F, Ismaelov A, de Haller R, et al. First-line tuberculosis therapy and drug resistant Mycobacterium tuberculosis in prisons. Lancet. 1999; 353(9157):969-73.

12. Dean JG, Dean JA, Coulombier D, Brendel KA, Smith DC, Burton AH, et al. Epi Info 6.0: a word processing database and statistics program for epidemiology on IBM compatible 
computers. Atlanta, Georgia: Centers for Disease Control and Prevention; 1994.

13. Jones TF, Craig AS, Valway SE, Woodley CL, Schaffner W. Transmission of tuberculosis in a jail. Ann Inter Med. 1999;131(8):557-63.

14. Drobniewski F. Tuberculosis in prisons - forgotten plague. Lancet. 1995;346(8980):948-9.

15. World Health Organization. Guidelines for the control of tuberculosis in prisons. Geneva: WHO; 1998

16. Hanau-Berçot B, Gremy I, Raskine I, Bizet J, Gutierrez MC, Boyer-Mariotte S, et al. A oneyear prospective study (1994-1995) for a first evaluation of tuberculosis transmission in French prisons. Int J Tuberc Lung Dis. 2000; 4(9):853-9.

17. Valway SE, Greifinger RB, Papania M, Kilburn JO, Woodley C, DiFerdinando GT, et al. Multidrug-resistant tuberculosis in the New York State prison system, 1990-1991. J Infect Dis. 1994;170(1):151-6.

18. Aerts A, Habouzit M, Mschiladze L, Malakmadze N, Sadradze N, Menteshashvili O, et al. Pulmonary tuberculosis in prisons of the ex-USSR state of Georgia: results of a nation-wide prevalence survey among sentenced inmates. Int J Tuberc Lung Dis. 2000; 4(12):1104-10.

19. São Paulo, Secretaria Estadual de Saúde. Divisão de Tuberculose, Centro de Vigilância
Epidemiológica e Centro de Referência e Treinamento, Doenças Sexualmente Transmissíveis/AIDS. Disponível em: www.cve.saude. sp.gov.br/htm/Sh todas.htm. Acessado em fevereiro de 2004.

20. Martin Sanchez V, Alvarez-Guisasola F, Cayla JA, Alvarez JL. Predictive factors of Mycobacterium tuberculosis infection and pulmonary tuberculosis in prisoners. Int J Epidemiol. 1995;24(3):630-6.

21. Zellweger JP, Coulon P. Outcome of patients treated for tuberculosis in Vaud County, Switzerland. Int J Tuberc Lung Dis. 1998;2(5): 372-7

22. Valway SE, Richards SB, Kovacovich J, Greifingeer RB, Crawford JT, Dooley SW. Outbreak of multi-drug-resistant tuberculosis in a New York State prison, 1991. Am J Epidemiol. 1994;140(2):113-22.

23. Oliveira HB, Moreira Filho DC. Treatment abandonment and tuberculosis recurrence: aspects of previous episodes, Brazil, 19931994. Rev Saude Publica. 2000;34(5):437-43.

24. Pablos-Méndez A, Sterling TR, Frieden TR. The relationship between delayed or incomplete treatment and all-cause mortality in patients with tuberculosis. JAMA. 1996;276(15): 1223-8.

25. Bakhshi SS, Hawker J, Ali S. Tuberculosis mortality in notified cases from 1989-1995 in Birmingham. Public Health. 1998;112(3): 165-8.

26. Frieden TR, Fujiwara PI, Washko RM, Hamburg MA. Tuberculosis in New York City turning the tide. N Engl J Med. 1995;333(4): 229-33.

27. Levy MH. Tuberculosis control practices in some prison systems of the Asia-Pacific Region. Int J Tuberc Lung Dis. 1999;3(9):769-73.

28. Rodrigo T, Caylà JA, Garcia de Olalla P, Brugal MT, Jansà JM, Guerrero R, et al. Effectiveness of tuberculosis control programmes in prisons, Barcelona 1987-2000. Int J Tuberc Dis. 2002;6(12):1091-7.

29. Martín V, Caylà JA, Bolea A, Castilla J. Mycobacterium tuberculosis and human immunodeficiency virus co-infection in intravenous drug users on admission to prison. Int $\mathrm{J} \mathrm{Tu}$ berc Lung Dis. 2000;4(1):41-6.

Manuscrito recebido em 27 de fevereiro de 2003. Aceito em versão revisada em 19 de novembro de 2003.

ABSTRACT Objective. To describe the incidence of tuberculosis among inmates of the municipal prison system in the city of Campinas, State of São Paulo, Brazil, from 1993 through 2000.

\section{Tuberculosis among city jail inmates in Campinas, São Paulo, Brazil}

Methods. A retrospective study was carried out of the number of cases of tuberculosis that were notified among inmates over the study period. This involved reviewing data from a total of 4293 inmates who were held in the four prisons that comprise Campinas' municipal prison system. Data were obtained from the tuberculosis database belonging to the Department of Social and Preventive Medicine of the School of Medical Sciences at the State University of Campinas.

Results. The incidence of tuberculosis peaked in 1994 (1 397.62 cases per 100000 ) and was lowest in 1999 (559.04 cases per 100 000). Most cases of tuberculosis occurred in inmates in the 25 to 34 year age group (62.6\%). Acquired immunodeficiency syndrome (AIDS) was the disease most commonly associated with tuberculosis (49.9\%), which was most often pulmonary in type (91.9\%), with positive sputum smears in $70.3 \%$ of the inmates tested. Most inmates had not received treatment before $(75.4 \%)$. The treatment dropout rate reached $49 \%$, and a cure was achieved in only $20.8 \%$ of the cases notified over the study period.

Conclusions. The incidence of tuberculosis observed in this study was moderate when compared to incidence rates found in other studies of prison inmates, but this could be due to underreporting. The frequency of treatment failure noted was low, since patients who were treated under supervision showed good adherence and were cured in the majority of cases. However, the high treatment dropout rate seen in the study is alarming because it could facilitate the spread of drug resistant strains of Mycobacterium tuberculosis to the community at large. Controlling tuberculosis among prison inmates should be part of routine interventions aimed at eliminating this disease from the community and at preventing resistance to antituberculosis therapy. 